PROCEEDINGS OF THE

AMERICAN MATHEMATICAL SOCIETY

Volume 128, Number 9 , Pages $2627-2636$

S 0002-9939(00)05272-2

Article electronically published on February 25, 2000

\title{
NEW UNIQUENESS THEOREMS FOR TRIGONOMETRIC SERIES
}

\author{
J. MARSHALL ASH AND SH. T. TETUNASHVILI
}

(Communicated by Christopher D. Sogge)

\begin{abstract}
A uniqueness theorem is proved for trigonometric series and another one is proved for multiple trigonometric series. A corollary of the second theorem asserts that there are two subsets of the $d$-dimensional torus, the first having a countable number of points and the second having $2^{d}$ points such that whenever a multiple trigonometric series "converges" to zero at each point of the former set and also converges absolutely at each point of the latter set, then that series must have every coefficient equal to zero. This result remains true if "converges" is interpreted as any of the usual modes of convergence, for example as "square converges" or as "spherically converges."
\end{abstract}

\section{Results}

\subsection{One dimensional results.}

Definition 1.1. Say that a trigonometric series

$$
\frac{a_{0}}{2}+\sum_{n=1}^{\infty} a_{n} \cos n x+b_{n} \sin n x
$$

converges absolutely at a point $x$ if $\sum_{n=0}^{\infty}\left|A_{n}(x)\right|<\infty$, where $A_{0}=\frac{a_{0}}{2}$ and for each positive integer $n, A_{n}(x)=a_{n} \cos n x+b_{n} \sin n x$.

Definition 1.2. Let $H$ and $\mathcal{D}$ be subsets of the torus $\mathbb{T}=[0,2 \pi)$. Say that the pair $(H, \mathcal{D})$ is uniquely determining for convergence if the only trigonometric series which converges to zero at each point of $H$ and converges absolutely at each point of $\mathcal{D}$ is the series which has all coefficients identically zero.

For example, Cantor's classical uniqueness theorem asserts that the only trigonometric series everywhere convergent to zero is the series which has all coefficients identically zero, or, equivalently, it asserts that $(\mathbb{T}, \varnothing)$ is uniquely determining. On the other hand, Menshov's trigonometric series which converges to zero almost everywhere shows that there is a set $E \subset \mathbb{T}$ of full (Lebesgue) measure, $|E|=2 \pi$, such that $(E, \varnothing)$ is not uniquely determining.

In 1937 Kozlov showed that a very small increase in the size of $\mathcal{D}$ allows a great decrease in the size of $H$ when he proved the following very interesting theorem [K].

Received by the editors August 1, 1998 and, in revised form, October 16, 1998.

1991 Mathematics Subject Classification. Primary 42A63, 42B99; Secondary 42A20, 42A24.

Key words and phrases. Trigonometric series, multiple trigonometric series, uniqueness. 
Theorem 1.1. There is a countable set $H$ and a set $\mathcal{D}$ with two elements such that $(H, \mathcal{D})$ is uniquely determining.

Definition 1.3. Let $H$ and $\mathcal{D}$ be subsets of $\mathbb{T}$ and let $T$ be a linear method of summability. Say that the pair $(H, \mathcal{D})$ is uniquely determining for the method $T$ if the only trigonometric series which is $T$ summable to zero at each point of $H$ and converges absolutely at each point of $\mathcal{D}$ is the series which has all coefficients identically zero.

In order to extend Koslov's theorem to higher dimensions, we found it necessary to first establish the following one dimensional generalization.

Theorem 1.2. Let $E \subset \mathbb{T}$ have full measure and let $\Delta$ be a nonempty open subset of $\mathbb{T}$. Then there is a countable set $H \subset E$ and a set $\mathcal{D} \subset \Delta \cap E$ with two elements such that $(H, \mathcal{D})$ is uniquely determining for any regular linear method of summability.

This theorem will be proved in Section 2 below.

Corollary 1.1. There is a countable set $H$ and a set $\mathcal{D}$ with two elements such that $(H, \mathcal{D})$ is uniquely determining for any linear method of summability.

Proof. Set $E=\Delta=\mathbb{T}$.

1.2. Higher dimensional results. We begin by defining a general mode of convergence for multidimensional series which will include several standard modes of convergence as special cases. Let the ordinary one dimensional trigonometric system on $\mathbb{T}$ be denoted as $T^{1}=\left\{t_{i}(x)\right\}_{i=0}^{\infty}$ where $t_{0}(x)=1$ and $t_{2 i-1}(x)=\cos i x$ and $t_{2 i}(x)=\sin i x$ for $i=1,2, \ldots$. Then define the $d$-multiple trigonometric system on $\mathbb{T}^{d}$ by $T^{d}=\left\{T_{n}(x)\right\}_{n \geq 0}=\left\{\prod_{j=1}^{d} t_{n_{j}}\left(x_{j}\right)\right\}_{n \geq 0}$, where $x=\left(x_{1}, \ldots, x_{d}\right) \in \mathbb{T}^{d}$, $n=\left(n_{1}, \ldots, n_{d}\right) \in \mathbb{Z}^{d}$, and $n \geq 0$ means that each $n_{i} \geq 0$. We will be discussing the $d$-multiple trigonometric series

$$
\sum_{n_{1}=0}^{\infty} \ldots \sum_{n_{d}=0}^{\infty} a_{n_{1}, \ldots, n_{d}} \prod_{j=1}^{d} t_{n_{j}}\left(x_{j}\right)=\sum_{n \geq 0} a_{n} T_{n}(x)
$$

For any $k \geq 0$ in $\mathbb{Z}^{d}$ we denote

$$
A_{k}(x)=\sum_{2 k-\mathbf{1} \leq n \leq 2 k} a_{n} T_{n}(x)
$$

where $\mathbf{1}=(1, \ldots, 1)$. Generically, the sum defining $A_{k}$ has $2^{d}$ terms, since each $n_{j}$ takes on each of the two values $2 k_{j}-1$ and $2 k_{j}$. However, in case some $k_{j}=0$, we interpret the corresponding inequality, $-1 \leq n_{j} \leq 0$, to mean that $n_{j}$ takes on only the value 0 . In defining our modes of convergence, we will follow the general usage and will always keep the terms in an $A_{k}$ grouped together. Thus, we will be considering $d$-multiple trigonometric series of the form

$$
\sum_{k \geq 0} A_{k}(x)
$$


Definition 1.4. The $d$-multiple trigonometric series (1.3) converges absolutely at the point $x$ if

$$
\sum_{k \geq 0}\left|A_{k}(x)\right|<\infty
$$

Let $\omega^{d}=\{\omega(N)\}_{N=0}^{\infty}$ be a sequence of finite subsets of $\left(\mathbb{Z}_{0}\right)^{d}$, where $\mathbb{Z}_{0}$ is the nonnegative integers. Say that this sequence belongs to the class $\Omega^{d}$ if

1. $\omega(0) \subset \omega(1) \subset \ldots \subset \omega(N) \subset \ldots$ and

2. $\bigcup_{N=0}^{\infty} \omega(N)=\left(\mathbb{Z}_{0}\right)^{d}$.

Let $S_{N}(x)$ be the following partial sum of the series (1.3):

$$
S_{N}(x)=\sum_{k \in \omega(N)} A_{k}(x) .
$$

Definition 1.5. The series (1.3) $\omega^{d}$ converges to the number $s$ at the point $x$ if $\lim _{N \rightarrow \infty} S_{N}(x)=s$.

Definition 1.6. If a mode of convergence is such that it can be represented by a sequence in $\Omega^{d}$, then say that it is an $\Omega^{d}$ mode of convergence.

Remark 1.1. It is easy to see that both square convergence and spherical convergence are examples of $\Omega^{d}$ modes of convergence.

Definition 1.7. Let $H$ and $\mathcal{D}$ be subsets of $\mathbb{T}^{d}$ and let $\omega^{d}$ be an $\Omega^{d}$ mode of convergence. Say that the pair $(H, \mathcal{D})$ is uniquely determining for the mode $\omega^{d}$ if the only trigonometric series which $\omega^{d}$ converges to zero at each point of $H$ and converges absolutely at each point of $\mathcal{D}$ is the series which has all coefficients identically zero.

Theorem 1.3. Let $d \geq 2$. Let $\mathcal{E} \subset \mathbb{T}^{d}$ have full measure, and let $\mathcal{O}$ be a nonempty open subset of $\mathbb{T}^{d}$. Then there is a countable set $H \subset E$ and a set $\mathcal{D} \subset \mathcal{O} \cap \mathcal{E}$ with $2^{d}$ elements such that $(H, \mathcal{D})$ is uniquely determining for every $\Omega^{d}$ mode of convergence.

This theorem will be proved for the case $d=2$ in Section 3 below. This is sufficient because the proof is purely inductive and no new ideas are required for the general case.

Corollary 1.2. There is a countable set $H$ and a set $\mathcal{D}$ with $2^{d}$ elements such that $(H, \mathcal{D})$ is uniquely determining for any $\Omega^{d}$ mode of convergence.

Proof. Set $E=\mathcal{O}=\mathbb{T}^{d}$.

Corollary 1.3. There is a countable set $H$ and a set $\mathcal{D}$ with $2^{d}$ elements such that $(H, \mathcal{D})$ is uniquely determining for each of the following modes of convergence: spherical, square, restricted rectangular, unrestricted rectangular, and one way iterated.

Since the case of one way iterated convergence requires a short separate argument, we have placed the proof of this corollary at the end of this paper.

Remark 1.2. Both of our main theorems have two aspects. First, that a determining pair $(H, \mathcal{D})$ exists with $H$ countable and $\mathcal{D}$ of cardinality $2^{d}$, and, second, that that pair can be selected subject to two constraints: that $H$ be contained in a preassigned 
set of full measure and that $\mathcal{D}$ be contained in a (small) preassigned open set. Since the first aspect is probably the more interesting, the corollaries have been stated in a simpler (although technically weaker) way, with all mention of constraints removed. Simpler proofs of the corollaries could be given by omitting the parts of the proofs of the main theorems that deal with satisfying the two constraints.

\section{Proof of the one Dimensional theorem}

Whenever $a$ and $b$ are real numbers selected from $\mathbb{T}, a+b$ will be interpreted to be addition modulo $2 \pi$. We will frequently make use of the fact that $\mathbb{T}$ is an abelian group under addition.

Definition 2.1. If $a \in \mathbb{T}$ and $E$ is a subset of $\mathbb{T}$, then $a+E=\{a+e: e \in E\}$.

Property 1. If $E \subset \mathbb{T}$ is any measurable set, then for any real number $a$ the set $a+E$ is also measurable and has the same measure.

Property 2. For any real number $t$ and any nonzero rational number $r$, the set $\{t+k r: k \in \mathbb{Z}\}$ is dense in $\mathbb{T}$.

Lemma 2.1. Let $E \subset \mathbb{T}$ have full measure. Let $\Delta \subset \mathbb{T}$ be a nonempty open interval. Then there exists a countable set $H \subset E$ and a set $\mathcal{D} \subset \Delta \cap E$ with cardinality $(\mathcal{D})=$ $\# \mathcal{D}=2$ such that if the series (1.1) converges absolutely at both points of $\mathcal{D}$, then the series (1.1) converges absolutely on all of $H$ also.

Proof. Let $\Delta=(\alpha, \beta)$ and let $r$ be a rational number such that

$$
0<r<\frac{|\Delta|}{4}
$$

By $\Delta^{\prime}$ we denote the interval concentric with $\Delta$ and having one-fourth its length. For any integer $k$ we denote

$$
E_{k}=k r+E
$$

By Property $1,\left|E_{k}\right|=|E|=2 \pi$ for each integer $k$, so $\left|\bigcap_{k \in \mathbb{Z}} E_{k}\right|=2 \pi$ also. Let

$$
t_{0} \in \Delta^{\prime} \cap \bigcap_{k \in \mathbb{Z}} E_{k}
$$

Then for any integer $k$, there is a $t_{k} \in E$ such that $t_{0}=t_{k}+k r$, whence

$$
t_{k}=(-k r)+t_{0} \text {. }
$$

Let

$$
H=\left\{t_{k}: k \in \mathbb{Z}\right\} .
$$

Since $t_{0} \in \Delta^{\prime}, t_{1}=(-r)+t_{0}$ also belongs to $\Delta$ and we may choose $\left\{t_{0}, t_{1}\right\}$ to be $\mathcal{D}$.

It remains to prove that if the series (1.1) converges absolutely on $\mathcal{D}$, then it also converges absolutely on $H$. We will use the following easily verified trigonometric identities:

$$
A_{n}(t+h)+A_{n}(t-h)=2 A_{n}(t) \cos n h \text { for all } t, h \in \mathbb{R} \text { and } n \in \mathbb{N} \text {, and }
$$

$$
A_{n}\left(t_{k}\right)=A_{n}\left(-k r+t_{0}\right)
$$


It follows from relations (2.6) and (2.7) that

$$
\begin{aligned}
A_{n}\left(t_{2}\right)+A_{n}\left(t_{0}\right) & =A_{n}\left(-2 r+t_{0}\right)+A_{n}\left(t_{0}\right)=2 A_{n}\left(-r+t_{0}\right) \cos n r \\
& =2 A_{n}\left(t_{1}\right) \cos n r .
\end{aligned}
$$

This allows us to deduce the absolute convergence of the series (1.1) at the point $t_{2}$ from the postulated absolute convergence at the points $t_{0}$ and $t_{1}$. The same argument extends the absolute convergence of $\sum A_{n}$ one step at a time, up and down the entire arithmetic progression $\left\{t_{k}\right\}=H$.

We now pass to the proof of Theorem 1.2

Proof. Let $M=\left[\alpha_{m n}\right]$ be any regular method of summability. Let $H=H(E, \Delta)$ and $\mathcal{D}=\mathcal{D}(E, \Delta)$ be the sets of Lemma 2.1 and assume that

$$
\lim _{m \rightarrow \infty} \sigma_{m}(t)=\lim _{m \rightarrow \infty} \sum_{n=0}^{\infty} \alpha_{m n} A_{n}(t)=0 \text { for each } t \in H
$$

and that

$$
\sum_{n=0}^{\infty}\left|A_{n}\left(t_{0}\right)\right| \text { and } \sum_{n=0}^{\infty}\left|A_{n}\left(t_{1}\right)\right| \text { are finite, where } \mathcal{D}=\left\{t_{0}, t_{1}\right\} \subset \Delta .
$$

We have

$$
\begin{aligned}
\sigma_{m}\left(t_{k}\right)+\sigma_{m}\left(t_{-k}\right) & =\sum_{n=0}^{\infty} \alpha_{m n}\left[A_{n}\left(-r k+t_{0}\right)+A_{n}\left(r k+t_{0}\right)\right] \\
& =2 \sum_{n=0}^{\infty} \alpha_{m n} A_{n}\left(t_{0}\right) \cos r k n
\end{aligned}
$$

and

$$
\sigma_{m}\left(t_{k+1}\right)+\sigma_{m}\left(t_{-k+1}\right)=2 \sum_{n=0}^{\infty} \alpha_{m n} A_{n}\left(t_{1}\right) \cos r k n .
$$

It follows from relations (2.9) and (2.11) that

$$
\lim _{m \rightarrow \infty} \sum_{n=0}^{\infty} \alpha_{m n} A_{n}\left(t_{0}\right) \cos r k n=0 \text { for all } k \in \mathbb{Z} .
$$

Define a function $f$ by the formula $f(x)=\sum_{n=0}^{\infty} A_{n}\left(t_{0}\right) \cos n x$. From the absolute convergence of $\sum A_{n}\left(t_{0}\right)$ follows the uniform convergence of the series defining $f$. Thus $f$ is a continuous function. But summability is consistent with convergence, so from relation (2.13) and Lemma 2.1] it follows that $f$ converges to 0 uniformly on $H$. By Property $2, H$ is dense in $\mathbb{T}$, so $f$ converges to 0 uniformly on all of $\mathbb{T}$. Thus $A_{n}\left(t_{0}\right)=0$ for each $n \geq 0$. A similar argument starting from $t_{1}$ instead of $t_{0}$ shows that $A_{n}\left(t_{1}\right)=0$ for each $n \geq 0$. In other words,

$$
a_{0}=0
$$

and for each $n \geq 1$ the following system of equations holds:

$$
\left\{\begin{array}{l}
a_{n} \cos n t_{0}+b_{n} \sin n t_{0}=0 \\
a_{n} \cos n t_{1}+b_{n} \sin n t_{1}=0
\end{array}\right.
$$


But the associated determinants,

$$
\left|\begin{array}{ll}
\cos n t_{0} & \sin n t_{0} \\
\cos n t_{1} & \sin n t_{1}
\end{array}\right|=\sin n\left(t_{1}-t_{0}\right)=\sin n r
$$

are all nonzero, whence for each $n \geq 1$, we have $a_{n}=b_{n}=0$.

\section{Proof of the two Dimensional theorem}

We will take $d=2$ and ease notation by writing $(x, y)$ for $\left(x_{1}, \ldots, x_{d}\right)$ and so on.

Lemma 3.1. Let $E$ and $E^{\prime}$ be subsets of $\mathbb{T}$ of full measure and let $\Delta$ and $\Delta^{\prime}$ be nonempty open subsets of $\mathbb{T}$. Let $\left(H_{1}(E, \Delta), \mathcal{D}_{1}(E, \Delta)\right)$ and $\left(H_{1}\left(E^{\prime}, \Delta^{\prime}\right), \mathcal{D}_{1}\left(E^{\prime}, \Delta^{\prime}\right)\right)$ be the corresponding pairs of sets produced by Lemma 2.1 above. Thus there are rational numbers $r$ and $r^{\prime}$ so that $H_{1}(E, \Delta)=\left\{x_{j}\right\}_{j \in Z}$, where $x_{j}=-r j+x_{0} \in E$, for each integer $j$ and $\mathcal{D}_{1}(E, \Delta)=\left\{x_{0}, x_{1}\right\} \subset E \cap \Delta$ and $H_{1}\left(E^{\prime}, \Delta^{\prime}\right)=\left\{y_{j}\right\}_{j \in Z}$, where $y_{j}=-r^{\prime} j+y_{0} \in E^{\prime}$, for each integer $j$ and $\mathcal{D}_{1}\left(E^{\prime}, \Delta^{\prime}\right)=\left\{y_{0}, y_{1}\right\} \subset E^{\prime} \cap \Delta^{\prime}$. If the series (1.3) converges absolutely on the 4 point set $\mathcal{D}_{1}(E, \Delta) \times \mathcal{D}_{1}\left(E^{\prime}, \Delta^{\prime}\right)$, then it also converges absolutely on all of $H_{1}(E, \Delta) \times H_{1}\left(E^{\prime}, \Delta^{\prime}\right)$.

Proof. Our hypothesis is that

$$
\sum_{m, n=0,0}^{\infty, \infty}\left|A_{m n}\left(x_{0}, y_{0}\right)\right| \text { and } \sum_{m, n=0,0}^{\infty, \infty}\left|A_{m n}\left(x_{1}, y_{0}\right)\right| \text { are finite }
$$

and

$$
\sum_{m, n=0,0}^{\infty, \infty}\left|A_{m n}\left(x_{0}, y_{1}\right)\right| \text { and } \sum_{m, n=0,0}^{\infty, \infty}\left|A_{m n}\left(x_{1}, y_{1}\right)\right| \text { are finite }
$$

where

$$
A_{m n}(x, y)=\sum_{k=2 m-1}^{2 m} \sum_{l=2 n-1}^{2 n} a_{k l} t_{k}(x) t_{l}(y)
$$

For any $x, y$, and $h$ we have

$$
A_{m n}(x+h, y)+A_{m n}(x-h, y)=2 A_{m n}(x, y) \cos m h
$$

and

$$
A_{m n}(x, y+h)+A_{m n}(x, y-h)=2 A_{m n}(x, y) \cos n h .
$$

Just as in the proof of Lemma 2.1 above, setting $h=r$ and using the first equation allows us to deduce the absolute convergence of $\sum A_{m n}$ at each point $\left(x_{i}, y_{0}\right)$ from condition (3.1) and also at each point $\left(x_{i}, y_{1}\right)$ from condition (3.2). Similarly, for each fixed $i$, setting $h=r^{\prime}$ and using the second equation, we can now deduce the absolute convergence at every $\left(x_{i}, y_{j}\right)$ from the just proved absolute convergence at the points $\left(x_{i}, y_{0}\right)$ and $\left(x_{i}, y_{1}\right)$.

Lemma 3.2. Let $p$ be any natural number and let $H$ be any infinite subset of $\mathbb{T}$. If

$$
\lim _{N \rightarrow \infty} \alpha_{N} \cos p t+\beta_{N} \sin p t=0 \text { for every } t \in H
$$

then

$$
\lim _{N \rightarrow \infty} \alpha_{N}=\lim _{N \rightarrow \infty} \beta_{N}=0
$$


Proof. Suppose that condition (3.6) does not hold. Then there is a subsequence on which $\rho_{N}=\sqrt{\left|\alpha_{N}\right|^{2}+\left|\beta_{N}\right|^{2}}$ is bounded away from zero. Restricting to this subsequence and replacing $\alpha_{N}$ and $\beta_{N}$ by $\frac{\alpha_{N}}{\rho_{N}}$ and $\frac{\beta_{N}}{\rho_{N}}$ respectively, shows that we may assume that the sequences $\left\{\alpha_{N}\right\}$ and $\left\{\beta_{N}\right\}$ are bounded. Now, without loss of generality, we may pass to a subsubsequence and assume that $\left\{\alpha_{N}\right\}$ tends to a nonzero limit $\alpha$. By extracting yet one more subsequence we may also assume that $\left\{\beta_{N}\right\}$ tends to a limit $\beta$. From condition (3.5) it follows that for each $t \in H$ there holds the equation $\alpha \cos p t+\beta \sin p t=0$, where $\alpha \neq 0$. This is a contradiction, since $H$ is infinite, but a nontrivial trigonometric polynomial of degree $2 p$ can have at most $2 p$ zeros on $\mathbb{T}$.

We now turn to the proof of Theorem 1.3.

Proof. We may assume that the given open set $\mathcal{O}$ is the open nonempty rectangle $\Delta \times \Delta^{\prime}$. For each $y \in \mathbb{T}$ let $\mathcal{E}_{y}=\{x \in \mathbb{T}:(x, y) \in \mathcal{E}\}$ denote the horizontal section of $\mathcal{E}$ at height $y$ and let $F=\left\{y \in \mathbb{T}:\left|\mathcal{E}_{y}\right|=2 \pi\right\}$. Apply Fubini's theorem to the integral of the characteristic function of $\mathcal{E}$ over $\mathbb{T}^{2}$ to see that

$$
|F|=2 \pi \text {. }
$$

Let $\left(H_{1}\left(F, \Delta^{\prime}\right), \mathcal{D}_{1}\left(F, \Delta^{\prime}\right)\right)$ be the pair of sets from Lemma 2.1 so that $H_{1}\left(F, \Delta^{\prime}\right)=$ $\left\{y_{j}\right\}_{j \in Z} \subset F$ and $\mathcal{D}_{1}\left(F, \Delta^{\prime}\right)=\left\{y_{0}, y_{1}\right\} \subset F \cap \Delta^{\prime}$. From the definition of $F$ and the former inclusion it follows that

$$
\left|\mathcal{E}_{y_{j}}\right|=2 \pi \text { for every } j \text {. }
$$

Letting $E=\bigcap_{j \in \mathbb{Z}} \mathcal{E}_{y_{j}}$ and applying condition (13.8) we see that

$$
|E|=2 \pi
$$

Again apply Lemma 2.1 to produce a pair of sets $\left(H_{1}(E, \Delta), \mathcal{D}_{1}(E, \Delta)\right)$ satisfying $H_{1}(E, \Delta)=\left\{x_{j}\right\}_{j \in Z} \subset E$ and $\mathcal{D}_{1}(E, \Delta)=\left\{x_{0}, x_{1}\right\} \subset E \cap \Delta$. Since $H_{1}(E, \Delta) \times$ $H_{1}\left(F, \Delta^{\prime}\right) \subset \mathcal{E}$ and $\mathcal{D}_{1}(E, \Delta) \times \mathcal{D}_{1}\left(F, \Delta^{\prime}\right) \subset \mathcal{E} \cap \mathcal{O}$, we must now show that $\left(H_{1}(E, \Delta) \times H_{1}\left(F, \Delta^{\prime}\right), \mathcal{D}_{1}(E, \Delta) \times \mathcal{D}_{1}\left(F, \Delta^{\prime}\right)\right)$ is uniquely determining for every $\Omega^{2}$ mode of convergence. So fix an $\Omega^{2}$ mode of convergence $\omega^{2}=\{\omega(N)\}_{N \in Z}$ and assume that $\sum A_{k}(x)$ satisfies

$$
\lim _{N \rightarrow \infty} S_{N}\left(x_{i}, y_{j}\right)=\lim _{N \rightarrow \infty} \sum_{(m, n) \in \omega(N)} A_{m n}\left(x_{i}, y_{j}\right)=0 \text { for every }(i, j)
$$

and that $\sum A_{k}(x)$ is absolutely convergent at all 4 points of $\mathcal{D}_{1}(E, \Delta) \times \mathcal{D}_{1}\left(F, \Delta^{\prime}\right)$. By Lemma 3.1 we know that

$$
\sum_{m, n=0,0}^{\infty, \infty}\left|A_{m n}\left(x_{i}, y_{j}\right)\right|<\infty \text { for every pair of integers }(i, j) .
$$

Fix a pair of indices $(\bar{i}, \bar{j})$. From the proof of Lemma 2.1 we know that there are two rational numbers $r$ and $r^{\prime}$ so that any point $\left(x_{i}, y_{j}\right)$ of $H_{1}(E, \Delta) \times H_{1}\left(F, \Delta^{\prime}\right)$ can be written as $\left(-i r+x_{0},-j r^{\prime}+y_{0}\right)$. From this there follow the identities

$$
y_{\bar{j}+k}=-r^{\prime} k+y_{\bar{j}} \text { and } y_{\bar{j}-k}=r^{\prime} k+y_{\bar{j}}, k=1,2, \ldots .
$$


From equation (3.4) we know that for any real $\tau$,

$$
S_{N}\left(x_{\bar{i}}, \tau+y_{\bar{j}}\right)+S_{N}\left(x_{\bar{i}},-\tau+y_{\bar{j}}\right)=2 \sum_{(m, n) \in \omega(N)} A_{m n}\left(x_{\bar{i}}, y_{\bar{j}}\right) \cos n \tau .
$$

Denote this sum as $Q_{N}(\tau)$. It follows from relation (3.11) that the sequence of continuous functions $\left\{Q_{N}(\tau)\right\}$ converges uniformly to a continuous function $f(\tau)$.

From conditions (3.10) and (3.11) we see that for each integer $k$ there holds

$$
\lim _{N \rightarrow \infty} S_{N}\left(x_{\bar{i}},-k r^{\prime}+y_{\bar{j}}\right)=\lim _{N \rightarrow \infty} S_{N}\left(x_{\bar{i}}, k r^{\prime}+y_{\bar{j}}\right)=0 .
$$

From conditions (3.13) and 3.14)

$$
f\left(k r^{\prime}\right)=\lim _{N \rightarrow \infty} Q_{N}\left(k r^{\prime}\right)=0 \text { for every integer } k,
$$

and so, taking Property 2, the density of $\left\{k r^{\prime}\right\}$, into account, we find that the continuous function $f$ must be identically zero. In other words,

$$
\lim _{N \rightarrow \infty} Q_{N}(\tau)=0 \text { for every } \tau \in \mathbb{T} \text {. }
$$

The uniformity of convergence of $Q_{N}$ to $f$ justifies the following interchange of order of integration:

$$
\begin{aligned}
\lim _{N \rightarrow \infty} \sum_{(m, \iota) \in \omega(N)} & A_{m \iota}\left(x_{\bar{i}}, y_{\bar{j}}\right) \\
= & \lim _{N \rightarrow \infty} \sum_{(m, n) \in \omega(N)} A_{m n}\left(x_{\bar{i}}, y_{\bar{j}}\right) \frac{1}{\pi} \int_{\mathbb{T}} \cos n \tau \cos \iota \tau d \tau \\
& =\frac{1}{\pi} \int_{\mathbb{T}}\left(\lim _{N \rightarrow \infty} \sum_{(m, n) \in \omega(N)} A_{m n}\left(x_{\bar{i}}, y_{\bar{j}}\right) \cos n \tau\right) \cos \iota \tau d \tau \\
& =\frac{1}{\pi} \int_{\mathbb{T}} f(\tau) \cos \iota \tau d \tau=0 \text { for every integer } \iota .
\end{aligned}
$$

By definition we have

$$
\begin{aligned}
A_{m \iota}(x, y) & =\sum_{k=2 m-1}^{2 m} \sum_{\ell=2 \iota-1}^{2 \iota} a_{k \ell} t_{k}(x) t_{\ell}(y) \\
& =A_{m}^{2 \iota-1}(x) \cos \iota y+A_{m}^{2 \iota}(x) \sin \iota y,
\end{aligned}
$$

where

$$
A_{m}^{2 \iota-1}(x)=\sum_{k=2 m-1}^{2 m} a_{k, 2 \iota-1} t_{k}(x) \text { and } A_{m}^{2 \iota}(x)=\sum_{k=2 m-1}^{2 m} a_{k, 2 \iota} t_{k}(x) .
$$

Substituting definition (3.17) into equation (3.16), for every integer $\iota$ we have

$$
\lim _{N \rightarrow \infty}\left[\left(\sum_{(m, \iota) \in \omega(N)} A_{m}^{2 \iota-1}\left(x_{\bar{i}}\right)\right) \cos \iota y_{\bar{j}}+\left(\sum_{(m, \iota) \in \omega(N)} A_{m}^{2 \iota}\left(x_{\bar{\imath}}\right)\right) \sin \iota y_{\bar{j}}\right]=0 .
$$

Since this equation, with $x_{\bar{i}}$ fixed, holds for every $y_{\bar{j}}$ from the infinite set $H_{1}\left(F, \Delta^{\prime}\right)$, we may apply Lemma 3.2 and conclude that

$$
\lim _{N \rightarrow \infty} \sum_{(m, \iota) \in \omega(N)} A_{m}^{2 \iota-1}\left(x_{\bar{i}}\right)=\lim _{N \rightarrow \infty} \sum_{(m, \iota) \in \omega(N)} A_{m}^{2 \iota}\left(x_{\bar{i}}\right)=0 \text { for each } x_{\bar{i}} .
$$


But from equation (3.11) it follows that

$$
\sum_{m=0}^{\infty}\left|A_{m \iota}\left(x_{\bar{i}}, y_{j}\right)\right|=\sum_{m=0}^{\infty}\left|A_{m}^{2 \iota-1}\left(x_{\bar{i}}\right) \cos \iota y_{j}+A_{m}^{2 \iota}\left(x_{\bar{i}}\right) \sin \iota y_{j}\right|<\infty .
$$

Without loss of generality we may assume that there are infinitely many positive integers $m_{j}$ such that

$$
\sum_{m=0}^{m_{j}}\left|A_{m}^{2 \iota-1}\left(x_{\bar{i}}\right)\right| \geq \sum_{m=0}^{m_{j}}\left|A_{m}^{2 \iota}\left(x_{\bar{i}}\right)\right| \text { for every index } j .
$$

From the density of $H_{1}\left(F, \Delta^{\prime}\right)$ there exists an integer $k$ so that

$$
\left|\cos \iota y_{k}\right|-\left|\sin \iota y_{k}\right| \geq \frac{1}{2} \text {. }
$$

Putting relations (3.20) and (3.22) together with the triangle inequality yields

$$
\begin{aligned}
\sum_{m=0}^{m_{j}} \mid A_{m}^{2 \iota-1}\left(x_{\bar{i}}\right) \cos \iota y_{j} & +A_{m}^{2 \iota}\left(x_{\bar{i}}\right) \sin \iota y_{j} \mid \\
& \geq\left|\cos \iota y_{j}\right| \sum_{m=0}^{m_{j}}\left|A_{m}^{2 \iota-1}\left(x_{\bar{i}}\right)\right|-\left|\sin \iota y_{j}\right| \sum_{m=0}^{m_{j}}\left|A_{m}^{2 \iota}\left(x_{\bar{i}}\right)\right| \\
& \geq\left(\left|\cos \iota y_{j}\right|-\left|\sin \iota y_{j}\right|\right) \sum_{m=0}^{m_{j}}\left|A_{m}^{2 \iota-1}\left(x_{\bar{i}}\right)\right| \\
& \geq \frac{1}{2} \sum_{m=0}^{m_{j}}\left|A_{m}^{2 \iota-1}\left(x_{\bar{i}}\right)\right| .
\end{aligned}
$$

Let $j \rightarrow \infty$ in inequality (3.23) and then use relation (3.20) to see that

$$
\sum_{m=0}^{\infty}\left|A_{m}^{2 \iota-1}\left(x_{\bar{i}}\right)\right|<\infty \text {. }
$$

From this and relations (3.21) we also have

$$
\sum_{m=0}^{\infty}\left|A_{m}^{2 \iota}\left(x_{\bar{i}}\right)\right|<\infty
$$

Now relation (3.19) amounts to

$$
\lim _{N \rightarrow \infty} \sum_{m \in \omega_{\iota}(N)} A_{m}^{2 \iota-1}\left(x_{\bar{i}}\right)=0,
$$

where we define $\omega_{\iota}(N)$ to be the section of $\omega(N)$ at height $\iota$. Since $\iota$ is fixed and $\omega^{2}=\{\omega(N)\} \in \Omega^{2}$, it is easy to see that $\left\{\omega_{\iota}(N)\right\}_{N=0,1, \ldots}$ is a one dimensional regular linear method of summability which we will call $T$. Since $\bar{i}$ is arbitrary, the one dimensional trigonometric series $\sum_{m=0}^{\infty} A_{m}^{2 \nu-1}(x)$ is summable $T$ to zero at each point of $H_{1}(E, \Delta)$ and, by relation (3.24), is also absolutely convergent on the same set. By Theorem 1.2 and definition (3.18), it follows that

$$
a_{m, 2 \iota-1}=0 \text { for every } m=0,1,2, \ldots
$$

In a similar way, replacing relation (3.24) with relation (3.25) and so on, we also have

$$
a_{m, 2 \iota}=0 \text { for every } m=0,1,2, \ldots
$$


But $\iota$ was an arbitrary nonnegative integer, whence $a_{m n}=0$ for all nonnegative $m$ and $n$, and our proof is complete.

It only remains to give the proof of Corollary [1.3.

Proof. See AW for definitions of the five modes of convergence that we have to consider. Spherical and square convergence can each be realized as an $\Omega^{d}$ mode of convergence by making an obvious choice for the sequence $\omega^{d}$, so the corollary follows immediately in these cases. Since both unrestricted rectangular convergence at a point and restricted rectangular convergence at a point imply square convergence at that point, the corollary for these two methods also follows immediately. There remains the case of one way iterated convergence.

Let $H$ and $\mathcal{D}$ be the pair of sets constructed in the proof of Theorem 1.3 . Suppose that the one way iterated $\operatorname{limit}_{\lim _{N \rightarrow \infty}}\left(\lim _{M \rightarrow \infty} \sum_{m=0}^{M} \sum_{n=0}^{N} A_{m n}(x, y)\right)$ is 0 for every $(x, y)$ in $H$ and that

$$
\sum_{m=0}^{M} \sum_{n=0}^{N}\left|A_{m n}(x, y)\right|<\infty
$$

at all 4 points of $\mathcal{D}$. By Lemma 3.1, it also follows that relation (3.26) holds at all points of $H$ as well. But it is easy to see that whenever a double numerical series is absolutely convergent, its one way iterated limit

$$
\lim _{N \rightarrow \infty}\left(\lim _{M \rightarrow \infty} \sum_{m=0}^{M} \sum_{n=0}^{N} a_{m n}\right)
$$

and its square $\operatorname{limit}_{\lim _{M \rightarrow \infty}} \sum_{m=0}^{M} \sum_{n=0}^{M} a_{m n}$ coincide. Thus $\sum \sum A_{m n}(x, y)$ is square convergent to 0 at each point of $H$. Now apply Theorem 1.3 .

We thank the referee for a number of corrections and useful suggestions.

\section{REFERENCES}

[AW] J. M. Ash and G. Wang, A survey of uniqueness questions in multiple trigonometric series, Harmonic Analysis and Nonlinear Differential Equations, A Volume in Honor of Victor L. Shapiro, Contemporary Math. 208(1997), 35-71. MR 99f:42019

$[\mathrm{K}] \quad \mathrm{V}$. Ya. Kozlov, On the connection between absolute convergence and the uniqueness of expansion of a function into trigonometric series, Doklady Akademii Nauk USSR, 15(1937), 417-420.

Department of Mathematics, DePaul University, Chicago, Illinois 60614-3504

E-mail address: mash@math.depaul.edu

Department of Mathematics, Georgian Technical University, Kortava St. 77, Tbilisi, Georgia 380075 